\title{
SARS-CoV-2, Uncontrolled Diabetes and Corticosteroids-An Unholy Trinity in Invasive Fungal Infections of the Maxillofacial Region? A Retrospective, Multi-centric Analysis
}

\author{
Aditya Moorthy ${ }^{1,2,3} \cdot$ Rohith Gaikwad $^{1,2} \cdot$ Shreya Krishna $^{2}$ (D) Raghuraj Hegde $^{4}$. \\ K. K. Tripathi ${ }^{5}$ Preeti G. Kale ${ }^{6}$ P. Subramanya Rao ${ }^{7,8}$ - Deepak Haldipur ${ }^{9}$. \\ Krishnamurthy Bonanthaya ${ }^{10}$
}

Received: 6 January 2021 / Accepted: 18 February 2021/Published online: 6 March 2021

(C) The Association of Oral and Maxillofacial Surgeons of India 2021

\begin{abstract}
Objectives Collate and analyse data of maxillofacial/rhinocerebro-orbital fungal infections reported during the era of the Covid-19 pandemic, with the aim of investigating the common contributing factors leading to such infections and of highlighting the significance of this surge seen in patients infected with SARS-CoV-2.

Method This retrospective observational multi-centric study analysed patient data collected from clinicians belonging to different specialties in Bangalore, India. The
\end{abstract}

Shreya Krishna

shreyakrishna249@gmail.com

1 Department of Oral and Maxillofacial Surgery, Trust-Well Hospital, Bangalore, India

2 Department of Oral and Maxillofacial Surgery, Rangadore Memorial Hospital, 1st Cross Rd, Shankarapuram, Basavanagudi, Bangalore, Karnataka 560004, India

3 Department of Oral and Maxillofacial Surgery, Apollo Hospitals, Bangalore, India

4 Opthalmic Plastic Surgery and Opthalmic Oncology Service, Department of Opthalmology, Manipal Hospital, Bangalore, India

5 School of Behavioural Sciences, National Forensic Sciences University, Gandhinagar, Gujarat, India

6 Department of Opthalmology, Trust-Well Hospital, Bangalore, India

7 Department of ENT, Rangadore Memorial Hospital, Bangalore, India

8 Department of ENT, Columbia Asia Hospital, Yeshwanthpur, Bangalore, India

9 Department of ENT, Trustwell Hospitals, Bangalore, India

10 Department of Maxillofacial Surgery, Bhagwan Mahaveer Jain Hospital, Bangalore, India data included the presentation and management of patients presenting with aggressive maxillofacial and rhino-cerebro-orbital fungal infections and explored the relationship between SARS-CoV-2, corticosteroid administration and uncontrolled diabetes mellitus.

Results All 18 patients were Covid positive. Sixteen of the 18 patients received steroids for Covid treatment and 16 patients were diabetic (of whom 15 patients who were diabetics received steroids for Covid-19 treatment). Loss of vision was noted in 12 of the 18 patients and 7 of them underwent orbital exenteration. The fungi noted was mucormycosis in 16 patients, aspergillosis in 1 patient and a mixed fungal infection in 1 patient. Eleven of the patients survived, 6 died and 1 was lost to follow-up. There was a significantly higher incidence of diabetes $(p=0.03)$ amongst these cohort of patients who were Covid-19 positive with mucormycosis. A significantly higher number $(p=0.0013)$ of patients were administered steroids at some point during the treatment.

Conclusion Despite the limited sample size, it is evident that there is a significant increase in the incidence of angioinvasive maxillofacial fungal infections in diabetic patients treated for SARS-CoV-2 with a strong association with corticosteroid administration.

Keywords Covid-19 - Mucormycosis · Rhino-cerebroorbital · Diabetes · Aspergillosis

\section{Introduction}

The health scenario of the latter part of 2019 and whole of 2020 has been dominated globally by a novel strain of severe acute respiratory syndrome coronavirus 2 (SARSCoV-2)—which, along with severe pneumonia, is also 
associated with strokes, venous thrombosis, renal failure, cardiomyopathy, coronary and systemic vasculitis $[1,2]$. Till date, it has afflicted over 72.3 million people worldwide with more than 1.61 million people succumbing to the disease. In India, 9.8 million people have been afflicted with over 143,000 people succumbing to it [3].

The predominant clinical phenotype of SARS-CoV-2 (Covid 19) manifests as an upper respiratory infection followed by pneumonia when the virus invades the respiratory epithelium binding to angiotensin converting enzyme 2 (ACE2) receptors. The more severe, second stage of this disease is caused by the systemic inflammation and coagulopathy causing direct damage to blood vessels, with hepatic, renal and cardiac injuries [4]. The coagulopathy seen is of complement-mediated thrombotic microangiopathies (TMA), rather than sepsis-induced coagulopathy or disseminated intravascular coagulation (DIC) [5]. This leads to endothelial damage and microvascular thrombosis. Besides diffuse alveolar damage with severe inflammatory exudation and coagulopathy, COVID-19 patients also have immunosuppression with a decrease in CD4 + T and CD8 + T cells [6].

In the backdrop of this COVID-19 expression, there has been a notable rise in the incidence of invasive fungal infections (IFI) of the maxillofacial region, namely mucormycosis and aspergillosis. Mucormycosis is a rare and ominous fungal disease caused by the order Mucorales fungi. The most common type of mucormycosis is the rhino-cerebro-orbital type which happens when spores are inhaled into the paranasal sinuses of susceptible hosts [7]. Tissue necrosis, often a late sign, is a hallmark of mucormycosis, resulting from angioinvasion and vascular thrombosis. It is a time-sensitive disease with the global case fatality rate being $46 \%$ [8]. Hosts susceptible to mucormycosis include diabetics, those on systemic corticosteroid use, patients with neutropenia and hematologic malignancies, stem cell transplant patients and immunocompromised individuals [9]. Aspergillosis invading into the sinuses is also seen in immunocompromised individuals. India has the second highest case burden of diabetes with $8.9 \%$ of adults, 77 million patients affected [10].

In the past 8 months, we have seen a manifold increase in the referrals for rhino-cerebro-orbital mucormycosis. We hypothesise that the immunocompromising effects of corticosteroids with microangiopathy of diabetes and possible peripheral microthrombi in COVID-19 provide the ideal host for mucormycosis, which due to its inherent angioinvasive nature leads to the increased incidence. This along with the high incidence of diabetes in our population and widespread use of corticosteroids as a part of treatment of COVID-19 seems to be causal to this increased incidence. Given the poor prognosis of this disease and its time liability, we aim to analyse our experience of 18 cases of IFI to draw correlations between causative factors to enable clinicians to understand an evolving disease pattern.

\section{Materials and Methods}

This is a retrospective observational, multi-centric analysis of eighteen cases of rhino-cerebro-orbital IFI treated between May 2020 and December 2020. The study was compliant with the Helsinki statement and is exempted by the Institutional ethics board due to its retrospective nature. Standard informed consent was obtained for all patients.

These patients were treated at various hospitals, by clinicians of three different specialties in Bangalore, India-ENT, Maxillofacial surgery and Ophthalmology. The patients presented with one or more of the following symptoms: facial cellulitis, maxillary sinusitis, headache, necrosis of palatal bone/mucosa or acute loss of vision (Figs. 1, 2, 3). These were either known cases of previous COVID-19 infection or their reverse transcriptase polymerase chain reaction (RT-PCR) COVID-19 test upon admission revealed that they were positive. All cases were treated with corticosteroids as a part of a standard blanket Covid 19 drug regime. Patients with a history of chemotherapy, radiotherapy, osteoradionecrosis, history of medication-related osteonecrosis of jaw, granulocytopenic patients or those on other immunomodulator drugs were excluded from the study.

Apart from ascertaining the current COVID-19 status, routine blood investigations and chest $\mathrm{X}$-ray were carried out, and any further investigations were based on specific patient needs. Computed tomography (CT) and/or magnetic resonance imaging (MRI) of the face (including orbits) and brain were performed for all cases (Fig. 4). Patient details are summarised in Table 1.

The presence of sinusitis with mucosal thickening of maxillary and ethmoid sinuses, orbital cellulitis, facial swelling, headache, proptosis, oedema of the extraocular

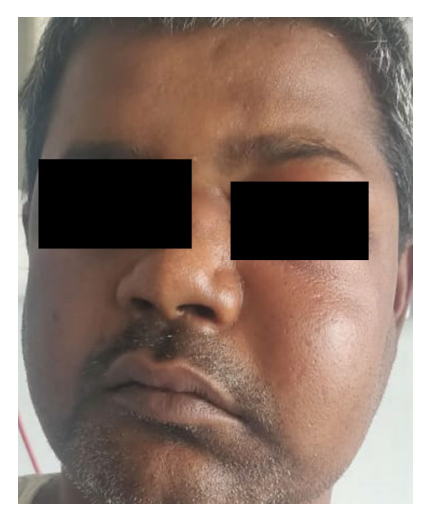

Fig. 1 Facial/orbital cellulitis in a patient with mucormycosis 


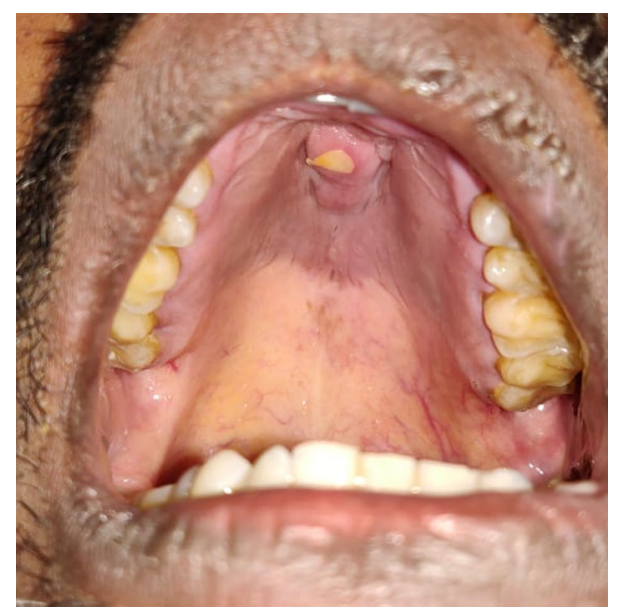

Fig. 2 Palatal mucosal discolouration in a patient with mucormycosis

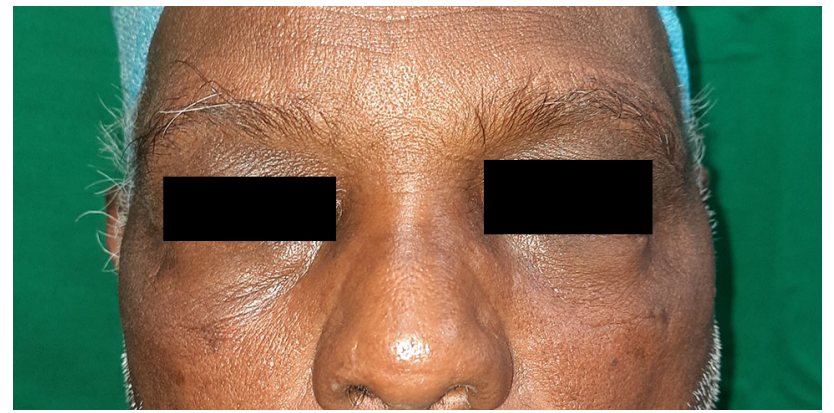

Fig. 3 Ptosis of left eye in a patient with frontal sinus mucormycosis

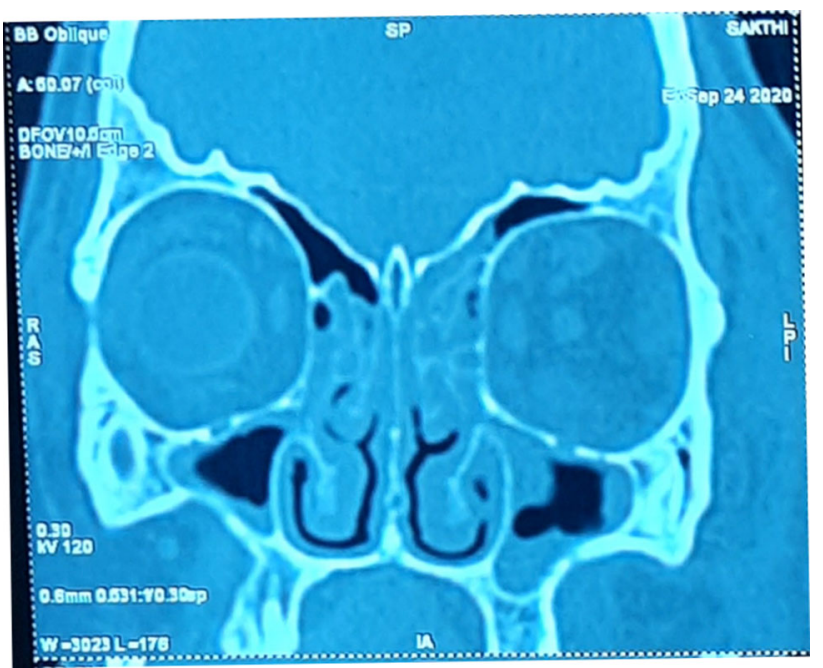

Fig. 4 Contrast enhanced computed tomography (CECT) images showing the involvement of left maxillary, ethmoid and frontal sinuses

muscles, ophthalmoplegia, etc. were few of the findings that led to a provisional diagnosis of invasive fungal infection. An ophthalmology referral was sought in patients suspected to have orbital extension of the disease.

Patients were assessed for the following before deciding the treatment protocol:

- History of COVID-19 infection/current COVID-19 infection

- Control of diabetes if patient was a diabetic (controlled/ poorly controlled)

- History of steroid use due to covid or administering of steroid if Covid detected at time of presentation

- Orbital involvement-acute/impending

- Intracranial extension

- Mortality

Patients were taken up for surgical exploration and debridement, assisted with sinus endoscopy and the infected tissue sent for a potassium hydroxide test ( $\mathrm{KOH}$ test) to determine the presence of fungal hyphae. The surgical intervention varied from a simple surgical debridement to maxillectomy (partial/subtotal/total) to orbital exenteration depending on the disease spread (Figs. 5, 6). $\mathrm{KOH}$ test was used for all cases as it was a rapid method of identifying the presence of fungal hyphae or yeast compared to the fungal culture which takes weeks for reporting. Tissue samples were also sent for fungal and bacterial culture as well as histopathologic examination. Once the $\mathrm{KOH}$ mount demonstrated fungal hyphae and the type of fungal element confirmed histologically (Fig. 7), depending on the organism, liposomal amphotericin therapy was instituted at $3-5 \mathrm{mg} / \mathrm{kg}$ for mucormycosis to a cumulative dose of between 3 and $5 \mathrm{~g}$. Oral voriconazole $(6 \mathrm{mg} / \mathrm{kg}$ IV q12hday $1 / 4 \mathrm{mg} / \mathrm{kg}$ IV q12h subsequently) and posaconazole (200 mg q8h) were administered for aspergillosis. Due to the possible nephrotoxicity of liposomal amphotericin B, daily renal function tests were performed. Alteration in dosage was tailored based on the renal tolerance. The patients were taken up for further surgical debridement if any suspicious lesions were noted on interval scans.

\section{Statistical Analysis}

To examine whether the distribution, determinants or sequels of the disease had a specific propensity, we calculated binomial probabilities using the equation

where $:=P(x=k)=\left(\begin{array}{l}n \\ k\end{array}\right) p^{k} q^{(n-k)}$

where 
Table 1 Patient determinants and distribution

\begin{tabular}{|c|c|c|c|c|c|c|}
\hline No & Age & $\operatorname{Sex}$ & Diagnosis & COVID status on admission & Diabetes & Steroid administration \\
\hline 1 & 58 & Male & Mucormycosis & Positive & Yes-poor control & Yes \\
\hline 2 & 56 & Male & Mucormycosis & Previously + ve & Yes-poor control & Yes \\
\hline 3 & 35 & Male & Mucormycosis & Previously + ve & Yes-poor control & Yes \\
\hline 4 & 73 & Male & Mucormycosis & Previously + ve & Yes-poor control & Yes \\
\hline 5 & 53 & Male & Mucormycosis & Previously + ve & Yes-poor control & Yes \\
\hline 6 & 55 & Male & Mucormycosis & Previously + ve & Yes-poor control & Yes \\
\hline 7 & 63 & Male & Mucormycosis & Previously + ve & Yes-poor control & Yes \\
\hline 8 & 52 & Female & Aspergillosis & Previously + ve & Yes-poor control & Yes \\
\hline 9 & 37 & Male & Mucormycosis & Positive & Yes-poor control & Yes \\
\hline 10 & 45 & Male & Mucormycosis + Aspergillosis & Positive & Non-diabetic & No \\
\hline 11 & 53 & Female & Mucormycosis & Previously + ve & Yes-poor control & Yes \\
\hline 12 & 68 & Male & Mucormycosis & Previously + ve & Yes-poor control & Yes \\
\hline 13 & 67 & Male & Mucormycosis & Previously + ve & Non-diabetic & Yes \\
\hline 14 & 61 & Male & Mucormycosis & Previously + ve & Yes-poor control & Yes \\
\hline 15 & 49 & Male & Mucormycosis & Previously + ve & Yes-poor control & Yes \\
\hline 16 & 63 & Female & Mucormycosis & Previously + ve & Yes-poor control & Yes \\
\hline 17 & 57 & Male & Mucormycosis & Positive & Yes-poor control & Yes \\
\hline \multirow[t]{6}{*}{18} & 39 & Male & Mucormycosis & Previously + ve & Yes-poor control & No \\
\hline & & Male-15 & Mucor-16 & Previously + ve -15 & Diabetic-16 & Yes-16 \\
\hline & & Female-3 & Aspergillus-1 & Positive on admn. -3 & Non-diabetic-2 & No-2 \\
\hline & & & Mixed-1 & & & \\
\hline & & $p=0.0075$ & $p=0.0309$ & & $p=0.0013$ (significant) & $p=0.0013$ (significant) \\
\hline & & (significant) & (significant) & & & \\
\hline
\end{tabular}

$$
\left(\begin{array}{l}
n \\
k
\end{array}\right)=\frac{n !}{k !(n-k) !}
$$

\begin{tabular}{ll}
\hline$k$ & Observed frequency of a success \\
$P$ & Probability of observed frequency $(x=k)$ \\
$p$ & Hypothesised probability of a success \\
$q$ & Hypothesised probability of not getting a success $(1-p)$ \\
$n$ & Number of trials \\
\hline
\end{tabular}

For example, to decide on the propensity of the disease to a specific gender, we calculated the probability of getting 0,1 or 2 male/female patients out of a total of 18 , had it been a chance occurrence. All these probabilities were added to determine the probability of such skewed occurrence of getting all such extreme values ( 3 or less male/ female patients) had it occurred by chance alone.

The above were exact probabilities and obviate the requirement of any interval estimate. Significance level was set at $p<0.05$. However, we have quoted exact probability of a particular (and equally extreme) occurrences.

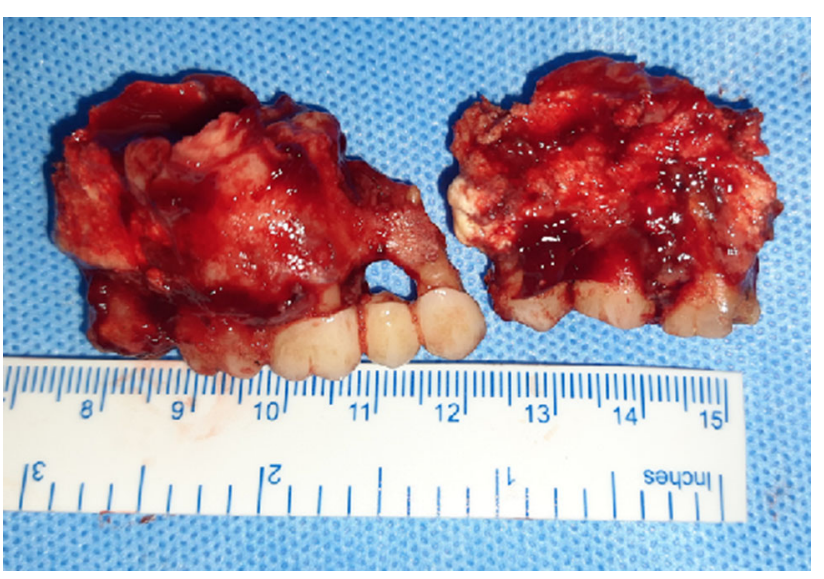

Fig. 5 Specimen of a partially resected maxilla in a patient who reported with avascular necrosis

\section{Results}

A total of 18 cases were included in the study, of which 15 were male and 3 were female. The age bracket of the patients was $35-73 y$ rs with a mean age of 54.6 years. All 18 patients were Covid positive, 14 previously and 4 on admission. Out of 18 patients, 16 were poorly controlled 
Fig. 6 Orbital exenteration done in a patient with loss of vision and orbital spread of the disease. A: Specimen of left eye along with contents of the orbit till the orbital apex. B: Left orbit after completion of exenteration
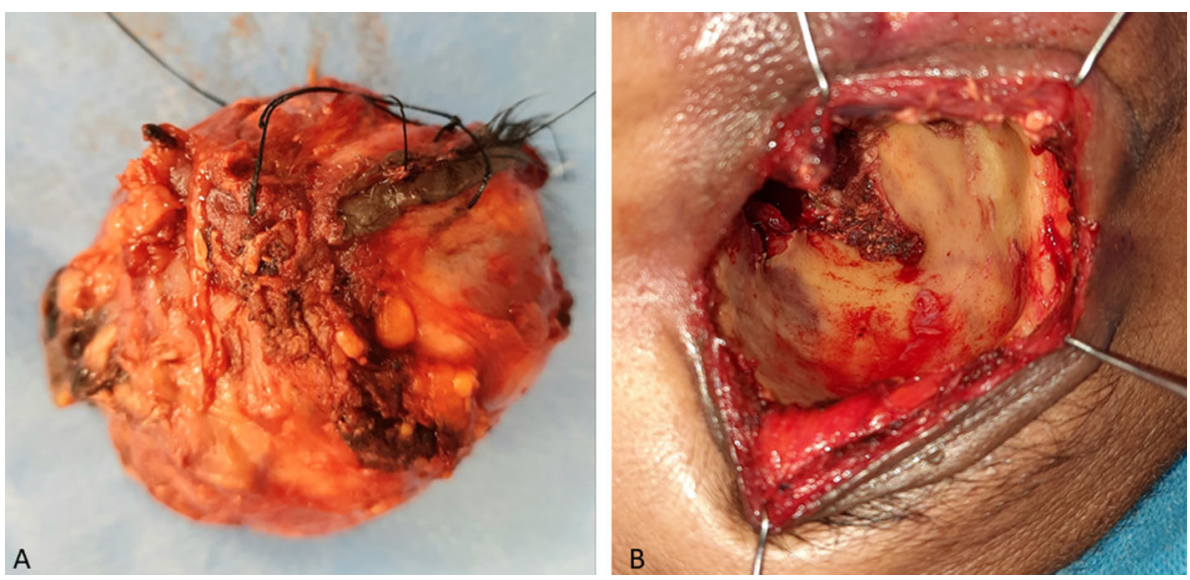

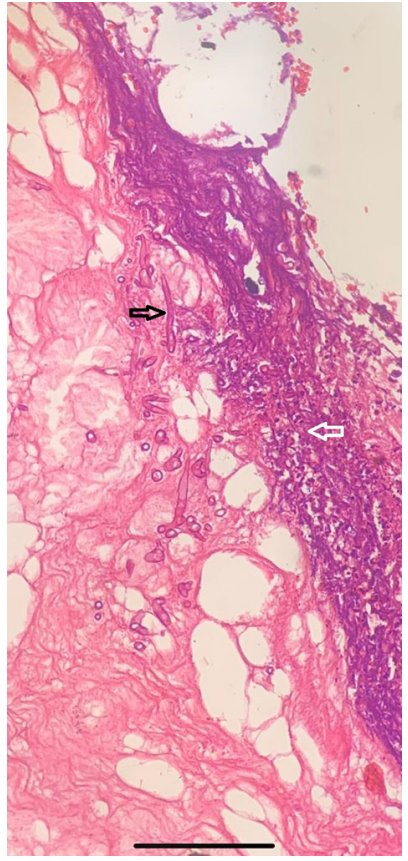

Fig. 7 Section shows broad aseptate hyphae branching at right angle (black arrowhead) consistent with mucor species ( $\mathrm{H}$ and $\mathrm{E} \times 40$ ) along with dense inflammatory infiltrate (white arrow)

diabetics and two patients were non-diabetics. All 18 patients presented with symptoms of maxillary sinusitis. Loss of vision was noted in 12 of the 18 patients (which was not statistically significant) and 7 of them underwent orbital exenteration (not statistically significant). Maxillary necrosis was seen in 14 of the patients (statistically significant; $p=0.03$ ), 11 of whom underwent maxillectomy (not statistically significant). Intracranial extension was seen in 9 patients (not significant statistically). All but two patients received steroids for management of their covid infection either previously or after diagnosis was confirmed upon admission. Endoscopic sinus surgery was performed in 17 patients. Mucormycosis was seen in 16 patients, aspergillosis in 1 patient and mixed infection in 1 patient.
All patients received liposomal amphotericin $\mathrm{B}$ at the dose of $3-5 \mathrm{mg} / \mathrm{kg}$. Eleven of the patients survived, 6 died and 1 was lost to follow-up. Of the deceased patients, $4 / 6$ had loss of vision at the time of presentation and 5/6 patients had intracranial extension. There was a significantly higher incidence of males in the study $(p=0.0075)$. There was a significantly higher incidence of diabetes $(p=0.03)$ amongst these cohort of patients who were Covid-19 positive with mucormycosis. A significantly higher number $(p=0.0013)$ of patients were administered steroids at some point during the treatment. Results are summarised in Table 2.

\section{Discussion}

Mucormycosis is a rare and lethal fungal infection caused by the family of Mucoraceae, which belongs to the class of Phygomycetes or Zygomycetes [11, 12]. This fungus usually resides as a commensal of the nasal mucosa and in conditions of immunosuppression like diabetes, ketoacidosis, solid organ transplant, severe burns, etc. can germinate in the nasal cavity and paranasal sinuses to invade the palate, orbits and brain, often leading to death [13]. The long-term use of glucocorticoids has also shown to contribute to mucormycosis in patients [14]. In this study, the authors observed a significantly high number of patients with a history of corticosteroid administration. The incidence of mucormycosis is not age or gender dependent, and the significantly higher number of males in this study may be a reflection of higher Covid-19 prevalence of males in India. In India till 25 May 2020, approximately $66.8 \%$ of Covid-19cases were identified to be males [15]. As mentioned earlier, the most common type of mucormycosis seen is rhino-cerebro-orbital mucormycosis (44-49\%), followed by cutaneous (10-19\%), pulmonary $(10-11 \%)$, disseminated (6-11\%) and gastrointestinal (2-11\%) [16]. Presenting signs and symptoms usually are: headache, 
Table 2 Sequelae, treatment and outcomes

\begin{tabular}{|c|c|c|c|c|c|c|c|c|}
\hline No & Loss of Vision & Sinusitis & Maxillary necrosis & $\begin{array}{l}\text { Intracranial } \\
\text { extension }\end{array}$ & FESS & Maxillectomy & Exenteration & Survival status \\
\hline 1 & Yes & Yes & Yes & Yes & Yes & Yes & Yes & Alive \\
\hline 2 & Yes & Yes & Yes & No & Yes & Yes & Yes & Alive \\
\hline 3 & Yes & Yes & Yes & Yes & No & No & Yes & Deceased \\
\hline 4 & Yes & Yes & Yes & Yes & Yes & No & No & Alive \\
\hline 5 & Yes & Yes & Yes & No & Yes & Yes & Yes & deceased \\
\hline 6 & Yes & Yes & No & No & Yes & No & No & Alive \\
\hline 7 & Yes & Yes & Yes & No & Yes & Yes & Yes & Alive \\
\hline 8 & Yes & Yes & No & Yes & Yes & No & No & Alive \\
\hline 9 & Yes & Yes & Yes & Yes & Yes & Yes & No & Deceased \\
\hline 10 & Yes & Yes & Yes & No & Yes & Yes & Yes & Alive \\
\hline 11 & Yes & Yes & Yes & Yes & Yes & Yes & Yes & Deceased \\
\hline 12 & No & Yes & Yes & Yes & Yes & Yes & No & Deceased \\
\hline 13 & No & Yes & No & Yes & Yes & No & No & Alive \\
\hline 14 & No & Yes & Yes & No & Yes & Yes & No & Alive \\
\hline 15 & No & Yes & No & Yes & Yes & No & No & deceased \\
\hline 16 & No & Yes & Yes & No & Yes & No & No & Survived \& LTF* \\
\hline 17 & Yes & Yes & Yes & No & Yes & Yes & No & Alive \\
\hline \multirow[t]{6}{*}{18} & No & Yes & Yes & No & Yes & Yes & No & Alive \\
\hline & & & $\begin{array}{l}\text { Maxillary necrosis- } \\
14\end{array}$ & Present-9 & & Yes-11 & Yes-7 & Alive -11 \\
\hline & & & $\begin{array}{l}\text { No maxillary } \\
\text { necrosis }-4\end{array}$ & Absent -9 & & $\mathrm{No}-7$ & No- -11 & Deceased-6 \\
\hline & & & & & & & & $\begin{array}{l}\text { *Lost to follow- } \\
\text { up-1 }\end{array}$ \\
\hline & $p=0.1189$ & & $p=0.0309$ & $p=0.2379$ & & $p=0.4807$ & $p=0.4807$ & \\
\hline & $\begin{array}{l}\text { NS (not } \\
\text { significant) }\end{array}$ & & Significant & NS & & NS & NS & \\
\hline
\end{tabular}

*LTF $=$ Lost to follow-up

fever, unilateral facial swelling, orbital cellulitis, with the presence of palpebral oedema, ptosis, chemosis and ophthalmoplegia [17]. The prognosis of the infection is poor with the overall mortality ranging between 33.3 and $80 \%$ $[17,18]$. Computed tomography (CT) is the first diagnostic tool used to assess status of sinuses, although extent of extra-sinus spread is best judged with magnetic resonance imaging (MRI) [11]. A definitive diagnosis of mucormycosis as the causative fungal species is achieved only after a histologic examination of the biopsy specimen is performed, and culture and $\mathrm{KOH}$ examination may be used only as a suggestive tool to identify the presence of mucormycosis. Aspergillosis of the Head and neck is also confirmed by histological examination.

The spurt of mucormycosis cases in recovered and fresh Covid-19 cases in multiple centres of Bangalore city, India, over the last 6 months is both a matter of concern and is clearly associated with treatment for the same with corticosteroids. The incidence (18 cases) that the authors have encountered in itself qualifies as an epidemic of mucormycosis within the Covid-19 pandemic. We have hypothesised this acute increase to be due to infection with Covid-19 contributing in more than one way. Firstly, the immune dysregulation seen as the reduced numbers of $\mathrm{T}$ lymphocytes, $\mathrm{CD} 4+\mathrm{T}$ and $\mathrm{CD} 8+\mathrm{T}$ cells may alter innate immunity increasing propensity for secondary fungal infections [19]. Secondly, the pathogenesis of Covid-19 resembles the spectrum of thrombotic microangiopathies (TMA) causing angioinvasion and endothelial damage much like that of mucormycosis, aggravating the disease [4]. Thirdly, glucocorticoids have been used extensively to reduce hospital stay and mortality related to Covid-19. Dexamethasone and methylprednisolone have both been incorporated into most protocols in COVID-19 infection especially in moderate to severe cases [20,21]. Due to the immunosuppressive nature of glucocorticoids, patients become more susceptible to secondary infections. 
The relationship between infection and diabetes is well established and bifacial. Uncontrolled diabetes creates a milieu for infections and infection derails glycaemic homeostasis [22]. People with type 2 diabetes are increasing in every country, but more than $80 \%$ live in low and middle -income countries such as India, Bangladesh, Bhutan, Pakistan, Sri Lanka, the Philippines and Indonesia. Amongst the top 10 countries in the world, India stands second with 77 million people with diabetes and another 36.5 million with prediabetes which is a high-risk condition for diabetes and cardiovascular disease [23]. As of 2019, the prevalence of diabetes in India has remained at $11.8 \%$ in the last four years, according to the National Diabetes and Diabetic Retinopathy Survey report, 2019. Males showed a similar prevalence of diabetes $(12 \%)$ as females $(11.7 \%)$. The association of mucormycosis with uncontrolled diabetes is proved and it is known to be an infection peculiar to diabetics, rarely seen in the normal population $[22,24]$. Diabetes in itself is not a risk factor for contracting Covid-19 infection; however, severe disease and adverse outcomes are more likely in individuals with diabetes. Conversely, Covid-19 infection can worsen diabetes control and some treatments used for COVID treatment (e.g. steroids) can exacerbate hyperglycaemia [22].

It is easy to assimilate from the above discussion that the increased incidence of mucormycosis and aspergillosis (IFI) of the maxillofacial region is not only caused by diabetes, Covid-19 infection and steroid administration independently, and this trinity has also coexisted in this pandemic and has self-potentiated the problem of immune dysregulation leading to fungal invasion and tissue necrosis. Our significant numbers of diabetics and steroid administration come to prove this very hypothesis. Due to the high mortality of the disease, early intervention by aggressive surgical debridement, systemic antifungals and management of underlying medical illness are quintessential to survival. A standard blanket protocol of steroid administration for Covid-19 infection may need to be revisited and an emphasis on tight glycaemic control during and after Covid-19 infection should be laid.

Central retinal artery occlusion (CRAO) is now a wellknown ophthalmic complication of Covid 19 that can lead to permanent vision loss [25-27]. Mucormycosis can cause both central ciliary and retinal artery occlusion [28]. In cases where blindness presents very late and there is clear radiological evidence of invasion of the orbital cavity by the mucor fungus, exenteration of the involved eye will help reduce gross disease burden and prevent intracranial spread. Some cases even warrant partial or total maxillectomy as seen in our study. Repeated surgical debridement may be needed for local control of the disease, and an aggressive surgical approach seems to improve patient survival. It is important to note that once the diagnosis is suspected, all immunosuppressive therapy should be reduced or discontinued if it is possible, and amphotericin $\mathrm{B} /$ voriconazole (depending on the organism isolated) is initiated [29].

\section{Conclusion}

SARS-CoV-2 has laid bare the limitations of human understanding of disease processes and its treatment. It is clear that a one-size-fits-all approach has significant limitations even in the public health setting. While corticosteroids have proved to be life-saving in several instances of Covid-19, it has proved to be a double edged sword and indiscriminate use has come with its price. It is vital that caution and discretion be used in the prescription of steroids. All patients should be monitored closely for sequelae of immunosuppression for months following treatment. The presence and severity of diabetes are an indicator of poor prognosis making good glycaemic control and early recognition vital for a favourable outcome. This ominous trinity of Covid-19 infection, diabetes and steroids makes it imperative that doctors have a low threshold of suspicion for this otherwise uncommon entity and act promptly albeit judiciously.

\section{Declarations}

Conflict of interest The authors have no relevant financial or nonfinancial interests to disclose. The authors have no conflicts of interest to declare that are relevant to the content of this article. All authors certify that they have no affiliations with or involvement in any organisation or entity with any financial interest or non-financial interest in the subject matter or materials discussed in this manuscript. The authors have no financial or proprietary interests in any material discussed in this article.

\section{References}

1. Guan WJ et al (2020) Clinical characteristics of coronavirus disease 2019 in China. N Engl J Med 382(18):1708-1720

2. Goyal P et al (2020) Clinical characteristics of Covid19 in New York City. N Engl J Med. https://doi.org/10.1056/nejmc2010419

3. COVID-19 Data Repository by the Center for Systems Science and Engineering (CSSE) at Johns Hopkins University)

4. Sweeny JM, Barouqa M, Krause GJ, Gonzalez-Lugo JD, Rahman S, Gil MR.Evidence for secondary thrombotic microangiopathy in COVID-19. medRxiv preprint https://doi.org/https://doi.org/ 10.1101/2020.10.20.20215608

5. Campbell CM, Kahwash R. Will complement inhibition be the new target in treating COVID-19 related systemic thrombosis? Circulation. 2020. [Epub ahead of print])

6. Peman J, Gaitan AR, Vidal CG, Salavert M, Ramirez P, Puchades F, Hita MG, Izquierdo AA, Quindo G (2020) Fungal co-infection in COVID-19 patients: should we be concerned? Rev Iberoam Micol 37(2):41-46

7. Cox G. 2020. Mucormycosis. Uptodate- July 2020. [Google Scholar]). 
8. Ehrenreich AW (2020) Mucormycosis with orbital compartment syndrome in a patient with COVID-19. Am J Emerg Med. https:// doi.org/10.1016/j.ajem.2020.09.032

9. Serris A, Danion F, Lanternier F (2019) Disease entities in mucormycosis. J Fungi 5(1):23

10. International Diabetes Federation. [Jul;2020 ]; https://idf.org/ournetwork/regions-members/south-east-asia/members/94-india. html

11. Ferguson BJ (2000) Mucormycosis of the nose and paranasal sinuses. Otolaryngol Clin North Am 33(2):2000

12. Uçkay I, Chalandon Y, Sartoretti P, Rhoner P, Berney T, Hadaya $\mathrm{K}$, van Delden C (2007) Invasive zygomycosis in transplant recipients. Clin Transplant 21:577e582

13. Mohindra S, Mohindra S, Gupta R, Bakshi J, Gupta SK (2007) Rhinocerebral mucormycosis:the disease spectrum in 27 patients. Mycoses 50:290e296

14. Gonzalez BDG, Garaa R, Gil F et al (2012) Mucormycosis of head and neck Report of five cases with different presentations. J Cranio Maxillo Facial Surg 40:584-591

15. Chanda A (2020) COVID-19 in India: transmission dynamics, epidemiological characteristics, testing, recovery and effect of weather. Epidemiol Infect 148:e243

16. Arnaiz-García ME, Alonso-Peña D, Gonzalez-Vela MC, GarcíaPalomo JD, Sanz-Gimenez- Rico JR, Arnáiz-García AM (2009) Cutaneous mucormycosis: report of five cases and review of the literature. J Plast Reconstr Aesthet Surg 62:434e441

17. Scheckenbach K, Cornely O, Hoffmann TK, Engers R, Bier H, Chaker A, Greve J, Schipper J, Wagenmann M (2010) Emerging therapeutic options in fulminant invasive rhinocerebral mucormycosis. Auris Nasus Larynx 37:322e328

18. Jung SH, Kim SW, Park CS, Song CE, Cho JH, Lee JH, Kim NS, Kang JM (2009) Rhinocerebral mucormycosis: consideration of prognostic factors and treatment modality. Auris Nasus Larynx 36:274e 279

19. Gangneux JP, Bougnoux ME, Dannaoui E, Cornet M, Zahar JR (2020) Invasive fungal diseases during COVID-19: we should be prepared. J Mycol Med 30:100971. https://doi.org/10.1016/j. mycmed.2020.100971
20. Clinical management protocol for COVID-19. (2020). Accessed: July7, 2020: https://www.mohfw.gov.in/pdf/Clin icalManagementProtocolforCOVID19.pdf

21. The Recovery Collaborative Group.Dexamethasone in Hospitalized Patients with Covid-19-Preliminary Report. N Engl J Med. July 17, 2020, at NEJM.org. http://doi.org/https://doi.org/10. 1056/NEJMoa2021436

22. Unnikrishnan R, Misra A (2020) Infections and diabetes: risks and mitigation with reference to India. Diab Metabol Synd Clin Res Rev 14:1889-1894

23. International Diabetes Federation (2015) IDF diabetes atlas, seventh edition. Brussels, Belgium: international Diabetes Federation

24. Rammaert B, Lanternier F, Poirée S, Kania R, Lortholary O (2012) Diabetes and mucormycosis: a complex interplay. Diab Metab 38(3):193-204

25. Montesel A, Bucolo C, Mouvet V, Moret E, Eandi CM (2020) Case report: central retinal artery occlusion in a COVID-19 patient. Front Pharmacol. https://doi.org/10.3389/fphar.2020. 588384

26. Acharya S, Diamond M, Anwar S, Glaser A, Tyagi P (2020) Unique case of central retinal artery occlusion secondary to COVID-19 disease. IDCases 21:e00867. https://doi.org/10.1016/ j.idcr.2020.e00867

27. Dumitrascu OM, Volod O, Bose S, Wang Y, Biousse V, Lyden PD (2020) Acute ophthalmic artery occlusion in a COVID-19 patient on apixaban. J Stroke Cerebrovasc Dis 29:2-4. https://doi. org/10.1016/j.jstrokecerebrovasdis.2020.104982

28. Luo QL, Orcutt JC, Seifter LS (1989) Orbital mucormycosis with retinal and ciliary artery occlusions. $\mathrm{Br} \mathrm{J}$ Ophthalmol 73(8):680-683. https://doi.org/10.1136/bjo.73.8.680

29. Spellberg B, Walsh TJ, Kontoyiannis DP, Edwards J Jr, Ibrahim AS (2009) Recent advances in the management of mucormycosis: from bench to bedside. Clin Infect Dis 48(12):1743e1751

Publisher's Note Springer Nature remains neutral with regard to jurisdictional claims in published maps and institutional affiliations. 